\title{
Perioperative Care of Adults with Congenital Heart Disease for Non-cardiac Surgery
}

\author{
Kimberly Howard-Quijano • Michelle Smith • \\ Johanna C. Schwarzenberger
}

Published online: 2 July 2013

(C) Springer Science + Business Media New York 2013

\begin{abstract}
In 2010 it was estimated that 1.2 million young adults in the United States have some form of congenital heart disease (CHD). Data from large adult congenital heart disease (ACHD) centers show that as many as $50 \%$ of these patients are over 40 years of age. As this population ages, physicians are likely to encounter such patients in their practice. Risk stratification and an interdisciplinary approach are paramount in achieving safe outcomes. The assessment of the patient must consider the variable expression of CHD. As most lesions are "fixed but not cured" the altered physiology of the heart and the physiological challenges of surgical interventions must be considered. The 2008 AHA/ACC guidelines for the Management of Adults with CHD establish for the first time a streamlined approach to the care of these patients. This review will apply these guidelines to the ACHD patient undergoing non-cardiac surgery.
\end{abstract}

Keywords Adult congenital heart disease .

Perioperative · General anesthesia - Heart failure .

Pulmonary hypertension · Eisenmenger

\footnotetext{
K. Howard-Quijano $(\bowtie) \cdot$ M. Smith · J. C. Schwarzenberger Department of Anesthesiology at the David Geffen School of Medicine, Ronald Reagan UCLA Medical Center, University of California, Los Angeles (UCLA), 757 Westwood Plaza, Suite 3325, Los Angeles, CA 90095, USA

e-mail: khquijano@mednet.ucla.edu

M. Smith

e-mail: mmsmith2@mednet.ucla.edu

J. C. Schwarzenberger

e-mail: jschwarzenberger@mednet.ucla.edu
}

\section{Introduction}

Diagnostic and therapeutic advancements in pediatric cardiology, cardiac surgery, anesthesiology, and intensive care have contributed to increased survival in patients with congenital heart disease (CHD). This has led to a shift in the population of CHD patients, with a rising number surviving to 40 years of age and beyond. Today, the number of adults with congenital heart disease (ACHD) exceeds children afflicted by the disease. As this population ages, they will likely present for non-cardiac procedures in increasing numbers. The American College of Cardiology (ACC) and American Heart Association (AHA) drafted guidelines detailing the desired management of ACHD, including recommendations for those undergoing non-cardiac surgery [1]. However this advice is based on level C evidence, partially due to the paucity of studies that examine the risks and outcomes of the ACHD patient undergoing non-cardiac interventions. This review aims to elucidate ways to risk stratify and coordinate the care of this diverse patient population.

\section{Peri Procedural Risk Assessment and Stratification}

While there are risk stratification guidelines for adult patients undergoing non-cardiac surgery, perioperative risk in adults with CHD is harder to evaluate. There is no uniform way of assessing cardiac function in the structurally altered heart with superimposed cardiac disease of adulthood. Only limited data is available on the perioperative risk stratification of ACHD patients undergoing noncardiac surgery. However, information on mortality and morbidity in ACHD offers some insight into the risks associated with this population. Price et al. [2] describes 
the altered outcome of a non-homogenous sample of ACHD patients undergoing low to high-risk non-cardiac surgery. While adult patients did not demonstrate a higher perioperative mortality, as reported in small infants, they were prone to higher resource utilization and longer intensive care unit (ICU) stays independent of the operative procedure. Using the national inpatient sample (NIS) data set, Rodriguez et al. [3••] investigated the prevalence of patients admitted to a US hospital in 2007 who carried the diagnosis of atrial septal defect (ASD) or ventricular septal defect (VSD). There were 84,308 admissions of adult patients who carried a diagnosis of CHD. Higher risk of inpatient mortality was seen in patients classified as young (18-30 years of age), or as very old ( $>50$ years of age). This suggests that the patients in the 30-50 year old group had selected themselves by having mild forms of septal defects without the added comorbidities of older age. Verheugt et al. [4] investigated the causes of mortality in this unique patient population by linking the Dutch national registry for ACHD patients with the national death registry between the years 2002-2009. They found excessive mortality associated with ACHD when compared to the general population. Heart failure (HF) and sudden death were the most common causes of mortality for both young adults and older patients. Predictors of mortality were; age, gender, complexity of the cardiac lesion, and number of interventions/complications. In addition, factors such as endocarditis, arrhythmias, pulmonary artery hypertension, conduction disturbances, and myocardial infarctions were predictors of worsening outcomes regardless of complexity of the cardiac lesion, age, and gender.

The 2008 ACC/AHA guidelines for the management of adults with $\mathrm{CHD}$ include a categorization of ACHD as low, moderate or high risk [1]. Low risk lesions are congenital cardiac lesions that are considered simple, as shown in Table 1. If corrected before the development of sequelae, lesions such as-patent ductus arteriosus (PDA), isolated atrial, and/or VSDs (without residual shunt) can be considered cured for life. Patients with simple CHD may have been "discharged" from their cardiologist and no longer receive follow-up. A detailed history regarding exercise tolerance, syncope, palpitations, episodes of dizziness or "blue spells" will determine whether referral to an ACHD cardiologist at a regional center is required. If these symptoms are not present, patients in the low risk category can receive standard perioperative care and do not require transfer to a specialized center.

Virtually all-remaining CHD lesions carry residual problems even after successful interventions that may result in progressive structural, contractile, hemodynamic, and electrophysiological changes of the heart. Patients in the moderate or high-risk category should be cared for at specialized centers, irrespective of the type of the
Table 1 Categorization of CHD lesion complexity and risk

Low risk
Small atrial septal defect
Isolated small ventricular septal defect (no associated lesions)
Small patent ductus arteriosus
Repaired patent ductus arteriosus, secundum or sinus venosus
atrial septal defect (without residua), or ventricular septal
defect (without residua)
Moderate risk
Anomalous pulmonary venous drainage, partial or total
Atrioventricular septal defects (partial or complete)
Coarctation (CoA) of the aorta
Ebstein's anomaly
Pulmonary valve regurgitation (moderate to severe)
Pulmonary valve stenosis (moderate to severe)
Valvular aortic stenosis (AS) or supra AS, except hypertrophic
obstructive cardiomyopathy (HOCM)
Tetralogy of fallot
Ventricular septal defect with other cardiac lesions
High risk
Conduits, valved or non-valved
Cyanotic congenital heart (all forms)
Double-outlet ventricle
Eisenmenger syndrome
Fontan procedure
Mitral atresia
Tringle ventricle (also called double inlet or outlet, common, or
Tricuspincus
Truncus arteriousus

procedure, see Table 1 . In these higher risk patients, endorgan dysfunction (CNS, lung, kidney) may result from repeated surgical interventions as well as from the longterm effects of cyanosis, polycythemia, cerebro-vascular accident, and pulmonary hypertension (PAH). In assessing patients pre-operatively, the most important consideration is the propensity for progression to a low cardiac output state, as well as, the presence of PAH, HF, cyanosis, conduction defects, or residual shunts. Patients with cyanotic CHD, especially with associated PAH are especially high risk.

A point system for the risk stratification of the CHD patient prior to undergoing a procedure was developed by Mossad (Table 2). A sum total of 0-6 points indicates minimal perianesthesia risk and interventions can be performed on an outpatient basis. A total of 6-13 points indicates moderate perianesthesia risk and procedures can possibly be performed with same day of admission, however transfer to a specialized $\mathrm{CHD}$ center may be 
Table 2 Point system to assess ACHD patient

\begin{tabular}{|c|c|c|c|}
\hline & 0 & 1 & 2 \\
\hline CHD diagnosis & Simple (ASD) & Moderate (ASD + PS) & Severe (TOF) \\
\hline CHD intervention & Corrected, no residual & Corrected with residual & Palliated \\
\hline Ventricular obstruction & None & Yes, gradient $<49 \mathrm{mmHg}$ & Yes, gradient $>50 \mathrm{mmHg}$ \\
\hline Ventricle, number, and position & $2 \mathrm{LV}$ is systemic ventricle & $1 \mathrm{LV}$ is systemic ventricle & $1 \mathrm{RV}$ is systemic ventricle \\
\hline Dysfunction systemic ventricle & Mild & Moderate & Severe \\
\hline PVR & Normal, $<2$ wood units & $2-4$ wood units & $>4$ wood units \\
\hline $\mathrm{SAO}_{2}$ & $>90 \%$ & $75-90 \%$ & $<75 \%$ \\
\hline Hct & $30-45 \%$ & $25-30 \%$, or $45-65 \%$ & $>65 \%$ \\
\hline Arrhythmias & Seldom & Atrial Level & Ventricular level \\
\hline No. of cardiac medications & 1 & 2 & $\geq 3$ \\
\hline
\end{tabular}

Add points for each line, and then assess risk according to total sum: 0-6 points, minimal peri-anesthesia risk, (outpatient), 6-13 points, moderate peri-anesthesia risk (possible same day admit), 14-20 points, high peri-anesthesia risk (possible ICU admit)

Modified from Mosad E, presented during Society of Cardiothoracic Annual meeting 2002

$A S D$ atrial septal defect, $P S$ pulmonary stenosis, $T O F$ tetralogy of fallot, $L V$ left ventricle, $R V$ right ventricle, $P V R$ pulmonary vascular resistance, $\mathrm{SAO}_{2}$ systemic oxygen saturation, $\mathrm{Hct}$ hematocrit

From Howard-Quijano and Schwarzenberger [27]. With permission from Wolters Kluwer Health

considered. High perianesthesia risk is indicated by a total of 14-20 points. Procedures for these patients should be performed in a specialized center and intensive care admission should be considered. Almost all patients in the high-risk (14-20 point) category will benefit from preoperative cardiac catheterization. The cardiac catheterization has the following goals: (1) the delineation of flow through the pulmonary $(Q \mathrm{p})$ versus flow through the systemic circulation $(Q \mathrm{~s})$ or in short $Q \mathrm{p}: Q \mathrm{~s},(2)$ documentation of valve regurgitation or obstruction, (3) measurements to discern volume loaded versus pressure loaded ventricle, and (4) assessment of PAH and testing of reactivity to pulmonary vasodilator therapy. Patients with residual lesions seen on cardiac catheterization, may undergo catheter-based intervention prior to planned non-cardiac surgery. Cardiac catheterization remains an excellent tool for targeted congestive HF therapy and possible electrophysiological testing and intervention (ablation/antitachycardia therapy, pacemaker, cardiac re-synchronization). Exercise stress testing (treadmill or stress echo) may be employed to assess cardiac function and may help determine how a patient will respond when challenged perioperatively with sudden volume or blood pressure changes.

\section{Arrhythmias}

CHD patients are at increased risk for developing arrhythmias due to chamber enlargement, surgical incisions, and fibrosis. These changes can be due to the intrinsic nature of the anomaly or changes in the myocardium associated with repair or palliation [5••, 6]. Increased frequency of arrhythmias occurs in those patients who underwent late repair for lesions such as ASD. Atrial fibrillation can be seen in CHD patients that are deemed low risk such as bicuspid aortic valve or mitral valve prolapse. Intra-atrial reentrant tachycardias are more commonly seen in patients with palliated lesions such as Fontan or atrial baffles for transposition of the great arteries (TGA). Ebstein's anomaly is associated with Wolfe-Parkinson's White syndrome in $20 \%$ of cases, most often when the valve is displaced in the posterior septal location [6]. Ventricular arrhythmias can also be seen in patients who have undergone a ventriculotomy (related to scar) or have right ventricular pressure overload. Right ventricular pressure overload is commonly seen in single ventricle patients with the right ventricle serving as the systemic ventricle, as well as, in patients with Eisenmenger syndrome or Ebstein's anomaly. Implantable cardiac defibrillators should be placed in patients with documented ventricular tachycardia (VT) or increased risk for VT. In addition, patients may require rate control, anticoagulation, or evaluation for cardiac thrombi and possible ablation of arrhythmic foci.

\section{Heart Failure}

Acquired HF in the general population is most often caused by coronary artery disease and ischemia, and there are well-established severity classification and treatment algorithms for this homogenous patient population. Classification and therapy of HF associated with ACHD is less well established. Patients with ACHD have been excluded from a majority of randomized controlled trials of treatments (i.e., beta blockers, angiotensin-converting enzyme 
inhibitors (ACE-I), angiotensin II receptor blockers (ARB)) for HF. It is unclear if the existing guidelines can serve the ACHD population [7, 8]. Many ACHD heart patients experience a combination of volume and pressure overload that cause the myocardium to fail. HF is more likely to occur in palliated hearts afflicted with Tetralogy of Fallot or hypoplastic left heart. Per the 2009 ACC/AHA guidelines on management of $\mathrm{HF}$, patients with ACHD are classified at least as stage B HF given structural heart disease even when asymptomatic [9].

Symptoms of HF in the ACHD population can be similar to those with acquired HF however, it may occur as early as the second or third decade of life. The neurochemical profile (increases in B-type natriuretic peptide, atrial natriuretic peptide, norepinephrine, and endothelin-1) in ACHD with HF is similar to those with acquired HF [7]. These markers mirror NYHA symptoms and ventricular function on echo. Symptoms alone may not be reliable for the diagnosis of HF in this population, as many ACHD patients in chronic compensated HF do not exhibit classic symptoms. Some studies have found a $49 \%$ sensitivity with using clinical judgment alone to diagnose HF [8]. Echo remains the standard for the evaluation of ventricular function. ICD placement for secondary prevention of sudden cardiac death should be considered in patients at risk for sudden death [8].

\section{Lesion Specific Management}

\section{Shunts}

\section{Atrial Septal Defect}

When the atrial septum does not form correctly, a communication or (ASD exists between the right and left atrium). There are five different types of ASDs: secundum, primum, sinus venosus, coronary sinus, and patent foramen ovale. ASDs make up 5-10\% of all congenital heart defects and secundum ASD's are most common, comprising nearly $80 \%$ of all ASD's [10]. A probe patent foramen ovale can be found in $\sim 30 \%$ of adult patients [11].

Atrial septal defects lead to a left to right shunt during diastole with the amount of shunting dependent on the size of the defect and the relative atrial pressures. This shunting can lead to right sided volume overload, pulmonary overcirculation, and PAH by the second or third decade of life. ASDs repaired prior to 12 years of age can be considered cured whereas repair beyond the age of 12 can lead to right-sided HF, frequent pulmonary infections, fatigue, exercise intolerance and atrial arrhythmias (atrial fibrillation/flutter/sick sinus). The longer an ASD remains unrepaired, the more likely the patient will develop sequelae
$[12,13]$. Patients with ASD's should be risk stratified based on the presence of these sequelae.

\section{Ventricular Septal Defect}

The ventricular septum is a muscular structure separating the right and left ventricles. Disruption of the ventricular septation causes a VSD resulting in communication between the two ventricles. There are four types of VSDs: subarterial (or supracristal), perimembranous (or infracristal), inlet (or canal type), and muscular [14•] A solitary VSD is a common congenital heart defect; however, VSDs also occur in $50 \%$ of complex CHD. A VSD can be seen in association with chromosomal abnormalities such as trisomy 13, 18, and 21 [15]. The inter-ventricular communication seen in unrepaired VSDs causes a left to right shunt at the ventricular level during systole. The amount of shunting is dependent on the size of the VSD and relative pressures in the right and left ventricles. If treated before $\sim 2$ years of age with no residual shunt, patients are likely to have normal cardiac physiology and function. If left untreated, left to right shunting will lead to volume overload in both the right and left ventricle with symptoms of PAH and HF often by 20 years of age $[16,17]$.

\section{Persistent Patent Ductus Arteriosus}

In fetal circulation, the ductus arteriosus shunts blood from the main pulmonary artery to the descending aorta. PDA is a physiologic shunt that closes functionally within $15 \mathrm{~h}$ of birth and should close permanently 2-3 weeks after birth. Closure normally occurs spontaneously as the result of increased oxygen concentrations and decreased circulating placental prostaglandins. Persistent PDA is a pathologic left to right shunt from the aorta to the main pulmonary that continues after birth [18]. The amount of shunting depends of the size, length and diameter of the PDA as well as the ratio of resistances between the pulmonary and systemic vasculature. A small shunt has little physiologic sequelae whereas large shunt causes increased pulmonary artery blood flow, left heart volume and pulmonary vascular resistance as well as pulmonary edema and eventual left HF if left untreated.

This increase in pulmonary vascular resistance can become irreversible, leading to PAH and right HF. If pulmonary vascular resistance becomes greater, then systemic vascular resistance can cause the shunt to reverse to right $\rightarrow$ left leading to cyanosis, chronic coronary underperfusion, respiratory infections and possible Eisenmenger syndrome [19]. A large PDA causes a decrease in aortic diastolic blood pressure due to backward flow of blood from the aorta to the PA leading to decreased coronary perfusion and myocardial ischemia. Untreated PDAs have 
a mortality rate of $\sim 1 / 3$ by 30 years of age and $2 / 3$ by 60 years of age [20॰]. A small PDA with minimal shunting can be closed percutaneously, often without long-term cardiovascular complications. Given the chronic over circulation of the lungs, they are at increased risk of acute respiratory distress syndrome (ARDS) following major surgery with large volume shifts.

\section{Recommendations}

Pre-operative evaluation in patients with unrepaired ASD, VSD or PDA should include echocardiography to determine the size of the communication, the degree and direction of shunting, and to evaluate for additional cardiac anomalies. Remaining intra-cardiac shunts have implications for surgical procedures that alter the pressure relationships in the heart (i.e., laparoscopy with $\mathrm{CO}_{2}$ insufflation) or are prone to intra-operative air/fat-embolus (craniotomy, orthopedic surgery). Management of ASDs and VSDs should focus on avoidance of hypovolemia and prevention of paradoxical embolism with air filters. Presence of PAH may trigger the use of a pulmonary artery catheter, nitric oxide, and external defibrillator peri-procedurally. For patients with VSDs, it is important to note that of the surgically corrected patients, $31 \%$ may have residual shunts, and, depending on the location of the VSD, may have complications of arrhythmias and atrioventricular block [14•]. It may also be imperative to close a PDA prior to proceeding with surgery, particularly if large volume shifts are anticipated.

\section{Valvular Disorders}

\section{Bicuspid Aortic Valve}

A normal aortic valve consists of three leaflets whereas a bicuspid aortic valve consists of two leaflets. This is the most common congenital cardiac malformation and seen in $\sim 1-2 \%$ of the population [21]. A bicuspid aortic valve can be associated with supravalvular or subvalvular obstruction and aortic coarctation. Adult patients with a bicuspid aortic valve are more likely to have premature coronary artery disease and aortic calcification or stenosis. A combination of turbulent flow and genetic alterations in tissue matrix can lead to aortic dilation [22, 23]. Aortic root dilation predisposes patients to aortic insufficiency, while dilation of ascending aorta increases the risk of aortic dissection.

Patients with bicuspid aortic valve should be evaluated pre-operatively for the presence of aortic stenosis, insufficiency, or aortic dilation. Management of patients with congenital aortic stenosis is similar to that of patients with acquired aortic stenosis. If needed, heart rate should be controlled with beta-blockers and patients should be maintained euvolemic with adequate afterload. Blood pressure should be evaluated in all four extremities to rule out aortic coarctation. Defibrillator pads should be placed on patients with congenital aortic lesions, as chest compressions are not as effective in patients with obstruction to left ventricular outflow or regurgitation leading to an inability to pressurize the aortic root and provide coronary perfusion. Patients with an aortic valve area of $<0.6 \mathrm{~cm}^{2}$, peak gradient of $>60 \mathrm{mmHg}$, left ventricular hypertrophy, or symptoms on exertion may benefit from aortic valve intervention prior to any non-cardiac surgery [21].

\section{Congenital Mitral Valve Disease}

The mitral valve is a bi-leaflet valve that regulates the flow of blood between the left atrium and left ventricle. The most common congenital mitral valve anomaly is mitral stenosis, in which there is obstruction to blood emptying from the left atrium into the left ventricle. This increase in left atrial pressures leads to increased left atrial size predisposing to arrhythmias and clot formation as well as pulmonary venous congestion. Mitral valve prolapse and cleft or parachute mitral valves can lead to mitral insufficiency, left atrial volume overload, and increased left atrial and pulmonary venous pressures [24].

Patients with congenital mitral valve disease should undergo pre-operative echocardiography to evaluate valve function and left atrial size and rule out other cardiac anomalies, left atrial thrombus, or PAH. Evaluation for arrhythmias can be made with a twelve lead electrocardiogram or a 24-h Holter monitor. If there is evidence of PAH or impaired left ventricular function, a pulmonary artery catheter may be considered and nitric oxide and external defibrillator pads should be readily available. Patients with congenital mitral stenosis can be managed similarly to patients with acquired mitral stenosis with heart rate control with beta blockade and maintenance of sinus rhythm, euvolemia and afterload.

\section{Congenital Pulmonary Valve Disease}

The pulmonary valve is tri-leaflet valve and controls the flow of blood from the right ventricle to the pulmonary artery. Congenital pulmonary valve disease can manifest itself as either incompetent leading to regurgitation, or stenotic causing an obstruction of blood flow from the right ventricle to the lungs. Congenital pulmonary valve disease can also be seen in conjunction with complex CHD or in association with an ASD [25]. All patients with congenital pulmonary valve disease should undergo pre-operative echocardiography to assess valve structure and function, as 
well to investigate any additional cardiac pathology. Balloon valvuloplasty is recommended if the pulmonary valve gradient is $>30 \mathrm{mmHg}$, and may be needed prior to an elective surgical procedure. Pulmonary insufficiency leads to regurgitation of blood from the pulmonary artery back into the right ventricle causing right ventricular volume overload/enlargement, arrhythmias, and possible right HF. Patients with severe pulmonary valve regurgitation may benefit from percutaneous pulmonary valve replacement prior to elective non-cardiac procedures.

\section{Infective Endocarditis}

ACHD patients are at increased risk for infective endocarditis if they have a history of: (1) prosthetic cardiac valve repair, (2) previous infective endocarditis, (3) unrepaired or palliated cyanotic heart disease, (4) completely repaired CHD with prosthetic material (catheter based or surgically) within the first six months following intervention, or (5) repaired CHD with residual defects preventing endothelization. In adult patients, infective endocarditis may be seen as frequently as in $1.5-6$ cases per 100,000 persons per year. Of these cases, $11-13 \%$ were also found to have CHD [26]. Interventions that increase the risk of endocarditis include dental cases involving the root or gingival and vaginal deliveries. Streptococci are the most frequent causative organisms in infective endocarditis except, in cases of cardiovascular device related infections, where staphylococci are more commonly associated.

The most recent recommendations for management of ACHD patients state that the diverse nature of CHD repairs and the paucity of scientific data make it difficult to provide definitive guidelines. Each patient should have treatment and care plans tailored to their individual clinical scenario [27]. General guidelines include prophylactic antibiotic treatment to high-risk ACHD patients undergoing high-risk dental or respiratory procedures, vaginal deliveries or hysterectomies. Recommended antibiotic prophylaxis for dental procedures includes oral regimen: amoxicillin (if penicillin allergic, azithromycin/clindamycin) and intravenous regimen: ampicillin or (if penicillin allergic clindamycin).

\section{Summary}

The variable nature of unaltered or surgically palliated CHD calls for an interdisciplinary approach for the ACDH patient undergoing non-cardiac surgery. "Fit for surgery" cannot be attested by a cardiologist alone. The anesthesiologist must work closely together with the entire team (cardiologist, surgeon, and/or interventionalists) to come up with a safe peri-procedure plan. The role of the cardiologist is to optimize the patient, prescribe SBE prophylaxis, and manage perioperative anti-coagulation or cardiac medications prior to surgery. The role of the surgeon is to assure for the most atraumatic and efficient intervention. The anesthesiologist must coordinate the care in the perioperative period and provide intraoperative care specific to the patient's lesion and risk, including potential need for invasive hemodynamic monitoring and transesophageal echocardiography. It is important that all caregivers convene prior to surgery and/or percutaneous intervention. Many patients have a prior history of difficult central and/ or peripheral intravenous access. In addition, if there is a history of prior Blalock-Taussig shunt, blood pressures may be different in their upper extremities. If no pulses can be palpated or if there is a history of central vein occlusion a pre-operative Doppler study is advisable. Patient disposition following the procedure needs as much planning as the peri-procedure conduct of anesthesia. Post-operative issues such as need for ventilation, or invasive monitoring, ICU admission and pain management should be addressed prior to the planned procedure. Managing the expectation of the patient and his/her family about their disposition is a key factor in the positive outcome of the ACHD patient.

In general, outpatient surgery is possible in the low- to moderate risk ACHD patient as long as the intervention is less invasive. Absence of pain and hemodynamic stability are prerequisite for discharge. ACHD patients undergoing more invasive procedures are high risk, and are better managed in a close observation unit. Close hemodynamic monitoring assures the patient's safety in the setting of preexisting altered cardiovascular physiology. For all practitioners involved in the care of this unique patient population it is imperative to know that the 2008 AHA guidelines encourage referral to a specialized center. Therapies such as oral pulmonary vasodilators (sildenafil, bosentan), electrophysiological intervention for arrhythmias, as well as, preventative strategies to avoid HF (afterload reduction with ACE inhibitors) may not only optimize the patient for the planned intervention but also positively influence the natural history of ACHD. In many instances, cardiac output can be enhanced by medical therapy (dobutamine, milrinone) allowing for emergency transfer of the high risk ACHD patient to a specialized center.

\section{Conclusion}

Low risk ACHD patients can safely undergo surgical and percutaneous intervention in non-specialized centers as long as the care team understands that few ACHD lesions are "cured" and the patient has undergone appropriate preoperative evaluation with characterization of the impact the 
patient's cardiac lesion may have on both their cardiac performance and peri-procedural course. High-risk ACHD patients should be referred to a specialized tertiary care center.

Disclosures Kimberly Howard-Quijano, Michelle Smith, and Johanna C. Schwarzenberger declare that they have no conflict of interest.

Compliance with Ethics Guidelines This article does not contain any studies with human or animal subjects performed by any of the authors.

\section{References}

Papers of particular interest, published recently, have been highlighted as:

- Of importance

-. Of major importance

1. Warnes CA, Williams RG, Bashore TM, et al. ACC/AHA 2008 guidelines for the management of adults with CHD: a report of the American College of Cardiology/American Heart Association Task Force on Practice Guidelines (Writing Committee to Develop Guidelines on the Management of Adults With CHD). Developed in collaboration with the American Society of Echocardiography, Heart Rhythm Society, International Society for Adult Congenital Heart Disease, Society for Cardiovascular Angiography and Interventions, and Society of Thoracic Surgeons. J Am Coll Cardiol. 2008;52:e1-121.

2. Price S, Jaggar SI, Jordan S, et al. Congenital heart disease: intensive care and outcome prediction. Intensive Care Med. 2007;33:652-9.

3. •• Rodriguez FH III, Moodie DS, Parekh DR, et al. Outcomes of hospitalization in adults in the United States with atrial septal defect, ventricular septal defect, and atrioventricular septal defect. Am J Cardiol. 2011;108:290-293. This is a comprehensive examination of nationwide inpatient data for patients with common forms of CHD to determine risk factors for morbidity and mortality. A discussion of the comorbidities that are commonly seen in this population includes a comparison with findings from other large studies.

4. Verheugt CL, Uiterwaal CS, van der Velde ET, et al. Mortality in adult congenital heart disease. Eur Heart J. 2010;31:1220-9.

5. - Seal R. Adult congenital heart disease. Pediatric Anesthesia. 21.5(2011):615-622. This is an excellent review on the management of ACHD patients undergoing cardiac and non-cardiac surgery, focusing on a multidisciplinary approach and describing an ideal model of care.

6. Lam W, Friedman R. Electrophysiology issues in adult congenital heart disease. Methodist DeBakey cardiovascular journal. 7.2(2011): 13

7. DiNardo JA. Heart failure associated with adult congenital heart disease. Seminars in cardiothoracic and vascular anesthesia. Vol. 17.No. 1.SAGE Publications, 2013.

8. Parekh DR. A review of heart failure in adults with congenital heart disease. Methodist DeBakey Cardiovasc J. 2011;7(2):26.

9. Hunt SA, Abraham WT, Chin MH, et al. Focused update incorporated into the ACC/AHA 2005 guidelines for the diagnosis and management of heart failure in adults: a report of the American College of Cardiology Foundation/American Heart Association Task Force on Practice Guidelines Developed in Collaboration with the International Society for Heart and Lung Transplantation. J Am Coll Cardiol. 2009;53(15):e1-90.

10. Rao PS. When and how should atrial septal defects be closed in adults. J Invasive Cardiol. 2009;21:76-82.

11. Krasuski RA, Hart SA, Allen D, et al. Prevalence and repair of intraoperatively diagnosed patent foramen ovale and association with perioperative outcomes and long-term survival. JAMA. 2009;302:290-7.

12. Webb G, Gatzoulis MA. Atrial septal defects in adults recent progress and overview. Circulation. 2006;114:1645-53.

13. Minette MS, Sahn DJ. Ventricular septal defects. Circulation. 2006;114:2190-7.

14. - Penny DJ, Vick WG. Ventricular septal defects. Lancet. 2011;377:1103-12. This is a comprehensive article on the pathophysiology, diagnosis and management of VSDs. It includes a table describing work-up, hemodynamic issues, indications for intervention and factors determining level of care in patients with repaired vs unrepaired VSDs.

15. Duffels MG, Engelfriet PM, Berger RM, et al. Pulmonary arterial hypertension in congenital heart disease: an epidemiologic perspective from a Dutch registry. Int J Cardiol. 2007;120:198-224.

16. Van de Bruaene A, Delcroix M, Pasquet A, et al. The Belgian Eisenmenger syndrome registry: implications for treatment strategies. Acta Cardiol. 2009;64:447-53.

17. Hamrick S, Hansmann G. Patent ductus arteriosus of the preterm infant. Pediatrics. 2010;125:1020-30.

18. Fortescue EB, Lock JE, Galvin T, et al. To close or not to close: the very small patent ductus arteriosus. Congenit Heart Dis. 2010;5:354-65.

19. Schneider DJ, Moore JW. Patent ductus arteriosus. Circulation. 2006; 114:1873-82.

20. - Siu SC, Silversides CK. Bicuspid aortic valve disease. J Am Coll Cardiol. 2010;55:2789-800. This article describes the genetics, pathology and clinical course of bicuspid aortic valve, as well as the medical management and indications for intervention.

21. Campbell M. The natural history of congenital aortic stenosis. Br Heart J. 1968;30:514-26.

22. Campbell M. Calcific aortic stenosis and congenital bicuspid aortic valves. Br Heart J. 1968;30:606-16.

23. Jiang $\mathrm{SJ}$, Gao $\mathrm{CQ}, \mathrm{Li} \mathrm{BJ}$, et al. Congenital mitral valve regurgitation in adult patients. Heart Surg Forum. 2011;14:114-6.

24. Andropoulos DB, Stayer SA, Russell IA, et al. Anesthesia for congenital heart disease. Massachusetts: Futura; 2005.

25. Bouza E, Menasalvas A, Munoz P, et al. Infective endocarditisa prospective study at the end of the twentieth century: new predisposing conditions, new etiologic agents, and still a high mortality. Medicine (Baltimore). 2001;80:298-307.

26. Wilson W, Taubert KA, Gewitz M, et al. Prevention of infective endocarditis: guidelines from the American Heart Association: aguideline from the American Heart Association Rheumatic Fever, Endocarditis, and Kawasaki Disease Committee, Council on Cardiovascular Disease in the Young, and the Council on ClinicalCardiology, Council on Cardiovascular Surgery and Anesthesia, and the Quality of Care and Outcomes Research Interdisciplinary Working Group. Circulation. 2007;116:1736-54.

27. Howard-Quijano K, Schwarzenberger JC. Acute care medicine: perioperative management of adult congenital heart disease. Am J Therap. 2012. doi:10.1097/MJT.0b013e3182456db9. 